\title{
MICELLIZATION BEHAVIOR COMPARISION OF POLYPEPTIDE GRAFT COPOLYMER AND BLOCK-GRAFT COPOLYMER IN ETHANOL
}

\author{
GUO-QUAN ZHU* \\ School of Materials Science and Engineering, Shandong University of Technology, Zibo 255049, P. R. China \\ (Received: December 2, 2009 - Accepted: May 19, 2010)
}

\begin{abstract}
Poly(ethylene glycol)-block-poly( $\gamma$-benzyl $L$-glutamate)-graft-poly(ethylene glycol) (PEG-b-PBLG-g-PEG) copolymer and poly( $\gamma$-benzyl $L$-glutamate)-graftpoly(ethylene glycol) (PBLG-graft-PEG) copolymer were synthesized by the ester exchange reaction of PEG chain with PBLG-block-PEG copolymer and PBLG homopolymer, respectively. The micellization behaviors of PEG- $b$-PBLG- $g$-PEG and PBLG-graft-PEG in ethanol were investigated by transmission electron microscopy (TEM) and viscometry. Effects of both the introduction of PBLG homopolymer and the change of testing temperature on the critical aggregation concentration (CAC) of the two polypeptide copolymers in ethanol were mainly studied. Nuclear magnetic resonance (NMR) spectroscopy and fourier transform infrared spectroscopy (FTIR) were used to research the chain conformations of polypeptide segments of the two polypeptide copolymers in solvent and in the solid state, respectively.
\end{abstract}

Keywords: Micellization, Morphology, Conformation, Critical aggregation concentration, Polypeptide copolymer

\section{INTRODUCTION}

Micellization behaviors of amphiphilic copolymers in selective solvents have received more attention both experimentally and theoretically ${ }^{1-14}$. Due to their amphiphilic characteristics, amphiphilic copolymers containing solvophobic and solvophilic components can self-assemble to form polymeric micelles or nanoparticles with a core-shell structrue ${ }^{1}$. The nanoscale structures possess a range of potential applications such as carriers of catalysts, drug delivery, protein simulation, macromolecular conformational study, nanoreactors etc $\mathrm{es}^{5-7}$.

Among the variety of self-assembled nanostructures, the polymeric micelles formed by block copolymers consisting of polypeptide segments and solvophilic polymer chains have received much interest for their great potential applications ${ }^{15-31}$. Cho et al. have reported the formation of polymeric micelles composed of poly( $\gamma$-benzyl $L$-glutamate) and poly(ethylene oxide) in aqueous medium and the drug delivery system based on the core-shell nanoparticles with PBLG as the hydrophobic inner core and PEO as the hydrophilic outer shell ${ }^{17}$. Harada et al. have studied the relationship between the conformation of the polypeptide chain and the supramolecular structure of poly( $L$-lysine)-blockpoly(ethylene glycol). It was revealed that the $\alpha$-helix structure of polypeptide segments tend to be stabilized by the PEG chains through the formation of a dimer with a micelle-like structure in aqueous medium ${ }^{18}$.

Relative to polypeptide block copolymers, polypeptide graft copolymers and polypeptide block-graft copolymers have received less attention. To the best of our knowledge, few experimental work has so far been reported on the micellization behavior comparison of polypeptide graft copolymer and polypeptide block-graft copolymer in ethanol. In this work, PEG- $b$-PBLG$g$-PEG copolymer and PBLG-graft-PEG copolymer were synthesized. The micellization behaviors of PEG- $b$-PBLG- $g$-PEG and PBLG-graft-PEG in ethanol were investigated by TEM and viscometry. Effects of the introduction of PBLG homopolymer and the change of testing temperature on the critical aggregation concentrations of the two polypeptide copolymers in ethanol were mainly studied. ${ }^{1} \mathrm{H}-\mathrm{NMR}$ spectroscopy and FTIR spectroscopy were used to research the chain conformations of polypeptide segments of the two polypeptide copolymers in solvent and in the solid state, respectively.

\section{EXPERIMENTAL}

\section{Materials}

The amine-terminated a-methoxy-w-amino poly(ethylene glycol) (ATPEG, $\left.M_{\mathrm{w}}=12,000\right)$ and poly (ethylene glycol methyl ether) (mPEG, $\left.M_{\mathrm{w}}=350\right)$ were purchased from Sigma Inc., and used without further purification. Hexane, tetrahydrofuran (THF) and 1,4-dioxane are of analytical grade and dried with sodium to remove water before use. All other solvents are of analytical grade and used without further purification.

Syntheses of polypeptide homopolymer and copolymers

The PBLG sample was prepared by a standard $N$-carboxyl- $\gamma$-benzyl- $L$ glutamate anhydride $\left(\gamma\right.$-BLG NCA) method ${ }^{1,32,33}$. The molecular weight of
PBLG was estimated from the intrinsic viscosity measured in dichloroacetic acid (DCA) ${ }^{34}$. The molecular weight of PBLG homopolymer used in the mixed system is 24,000 , while the molecular weight of PBLG homopolymer used in the ester exchange reaction is 90,000 . The PBLG-graft-PEG copolymer (shown as Figure 1a) was prepared by the ester exchange reaction of PBLG homopolymer with $\mathrm{mPEG}$ in 1,2-dichloroethane with $p$-toluenesulfonic acid as a catalyst according to the method described in the documents $\mathrm{s}^{1,35,36}$. Calculations, according to the results of the NMR measurements (Avance 550 ), present that the grafting ratio of PBLG-graft-PEG is about $21.4 \%$, and the molecular weight $\left(M_{\eta}\right)$ of PBLG-graft-PEG is about 112,000.

PBLG-block-PEG copolymer was obtained by the ring-opening polymerization of $\gamma$-BLG NCA initiated by AT-PEG in 1,4-dioxane at room temperature ${ }^{15}$. PEG- $b$-PBLG- $g$-PEG copolymer (shown as Figure 1b) was obtained by the ester exchange reaction of PBLG-block-PEG with mPEG in 1,2-dichloroethane with $p$-toluenesulfonic acid as a catalyst according to the method described in the literatures ${ }^{35,36}$. The grafting ratio of PEG- $b$-PBLG$g$-PEG copolymer was estimated by nuclear magnetic resonance (NMR) measurements (Avance 550). It was calculated by the peak intensities of the methylene proton signal $(5.08 \mathrm{ppm})$ of polypeptide and the ethylene proton signal (3.63 ppm) of PEG in the ${ }^{1} \mathrm{H}-\mathrm{NMR}$ spectrum ${ }^{35,36}$. To emphasize one point, for PEG- $b$-PBLG- $g$-PEG copolymer, the peak area $\left(\mathrm{A}_{\mathrm{d}-3.63}\right)$ used in calculating the grafting ratio is the subtraction of $A_{1}$ and $A_{2}\left(A_{1}>A_{2}\right)$, where $\mathrm{A}_{1}$ is the peak area $(3.63 \mathrm{ppm})$ in PEG- $b$-PBLG- $g$-PEG spectrum, $\mathrm{A}_{2}$ is the peak area (3.63 ppm) in PBLG-block-PEG spectrum. According to the ${ }^{1} \mathrm{H}-\mathrm{NMR}$ analysis, the grafting ratio of PEG- $b$-PBLG- $g$-PEG is about $20.6 \%$. The molecular weights $\left(M_{\eta}\right)$ of PBLG-block-PEG and PEG- $b$-PBLG- $g$-PEG are about 120,000 and 136,000 , respectively. The schematic representations of PBLG-graft-PEG copolymer structure and PEG- $b$-PBLG- $g$-PEG copolymer structure are presented in Figure 2.

Preparation of polypeptide copolymer micelles

The micelles of the polypeptide copolymer were prepared according to the document ${ }^{13}$. The obtained polypeptide copolymer sample was first dissolved in $\mathrm{CHCl}_{3}$ to make a $2 \mathrm{~g} / 1$ polymer solution. Subsequently, a given volume of ethanol was added into the polymer $\mathrm{CHCl}_{3}$ solution with stirring. The formation of the polypeptide copolymer micelles occurred, as indicated by the appearance of turbidity in the solution, when about $20 \mathrm{vol} \%$ ethanol was added. After 2-3 $\mathrm{h}$, the addition of ethanol was continued until the polymer concentration in the micelle solution was about $0.2 \mathrm{~g} / 1$. The micelle solution was kept overnight and then dialyzed against ethanol using dialysis membranes (3500 molecular weight cut-off) to remove the $\mathrm{CHCl}_{3}$ for $48 \mathrm{~h}$ at room temperature. It was preferred that ethanol was exchanged at intervals of 10-12 h. The solution was diluted with ethanol to the desired concentration.

${ }^{1} \mathrm{H}-\mathrm{NMR}$ measurements

${ }^{1} \mathrm{H}-\mathrm{NMR}$ spectra of polypeptide copolymers were measured in $\mathrm{CDCl}_{3}$ using a NMR instrument (Avance 550) at $500 \mathrm{MHz}$.

FTIR measurements

FTIR spectra of solid films of the polypeptide copolymer samples cast from chloroform solution were measured on a Nicolet 5700 FT-IR spectrometer 
between 4,000 and $400 \mathrm{~cm}^{-1}$.

Observation of transmission electron microscope

The morphology of the micelles was obtained by TEM (JEM-1200-EXII). Drops of micelle solution were placed on a carbon film coated copper grid, and then were dried at room temperature. Before the observations, the sample was stained by aqueous phosphotungstic acid solution $(1.0 \mathrm{wt} \%)$. The TEM bright field imaging was performed with $120 \mathrm{kV}$ accelerating voltage.

\section{Viscosity measurements}

Viscosity measurements of the micelle solution were made in an Ubbelohde viscometer, which was placed in a thermostatically controlled bath with a precision of $\pm 0.1^{\circ} \mathrm{C}$. The measurements were repeated at least three times and the times obtained were arithmetically averaged, then converted to the relative viscosity $\left(h_{\mathrm{g}}\right), h_{\mathrm{g}}$ was further converted to the specific viscosity $\left(h_{\mathrm{sp}}\right)$. The experiments were carried out by diluting the micelle solution step by step. The curve of $h_{\mathrm{sp}} / \mathrm{C}$ versus the concentration (C) of the micelle solution was drawn. By analyzing the curve of $h_{\mathrm{sp}} / \mathrm{C} \sim \mathrm{C}$, the critical aggregation concentration of polypeptide copolymers could be obtained ${ }^{14}$.
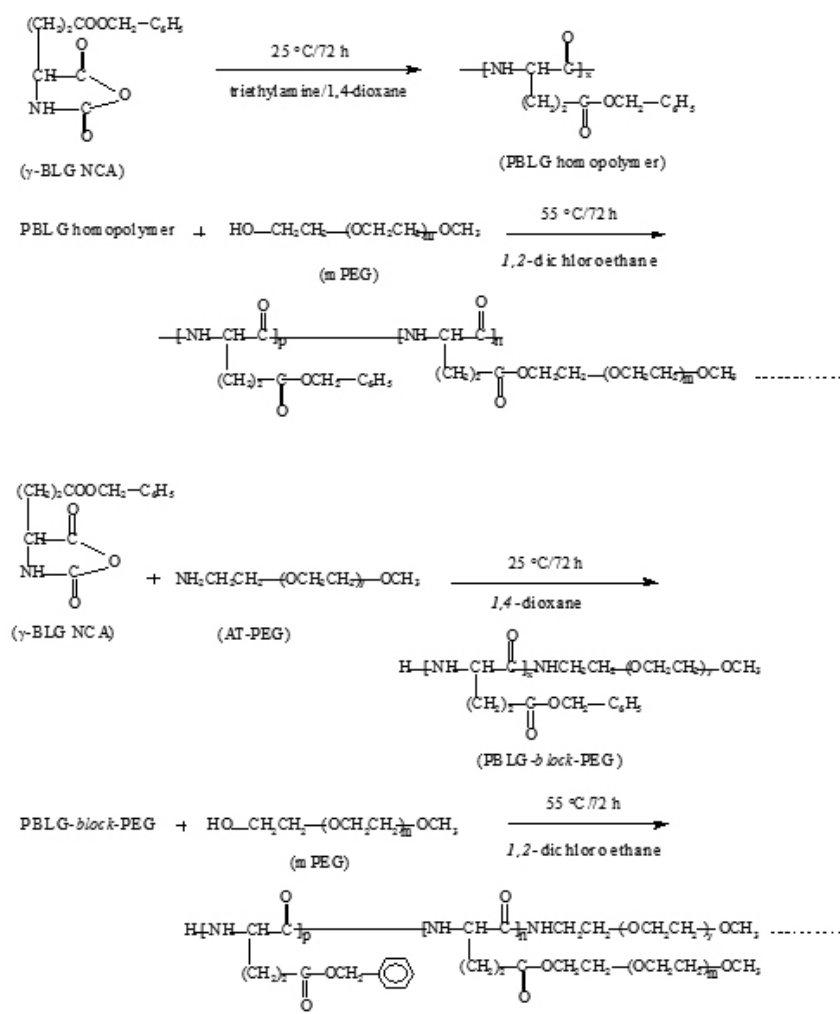

Figure 1. Synthesis of (a) PBLG-graft-PEG and (b) PEG- $b$-PBLG-g-PEG.
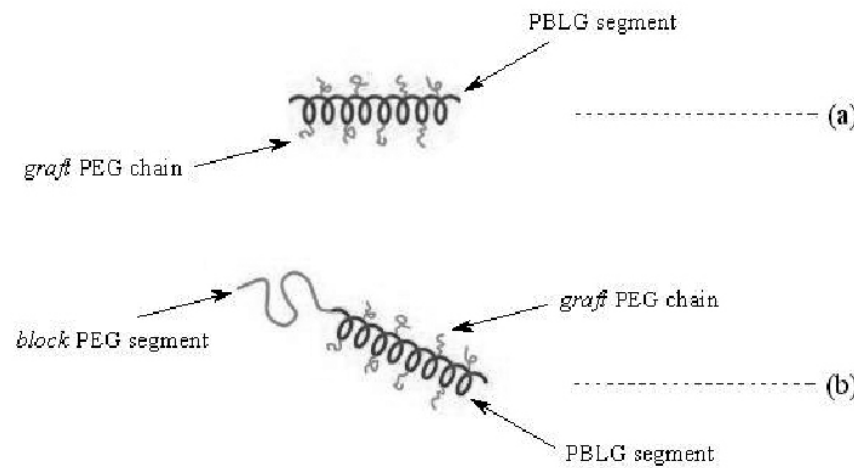

Figure 2. Schematic representations of (a) PBLG-graft-PEG copolymer structure and (b) PEG- $b$-PBLG- $g$-PEG copolymer structure.

\section{RESULTS AND DISCUSSION}

Chain conformation of polypeptide segments in the polypeptide copolymers dissolved in $\mathrm{CDCl}_{3}$

Figure 3 shows the ${ }^{1} \mathrm{H}-\mathrm{NMR}$ spectra of (a) PBLG-graft-PEG copolymer and (b) PEG- $b$-PBLG- $g$-PEG copolymer in $\mathrm{CDCl}_{3}$. As is shown in Figure 3 , for both PBLG-graft-PEG and PEG- $b$-PBLG- $g$-PEG, the characteristic peaks appearing at about $8.35 \mathrm{ppm}$ (corresponding to $\mathrm{NH}$ protons of polypeptide segments in the two polypeptide copolymers) are detected. This phenomenon demonstrates that polypeptide segments in both PBLG-graft-PEG copolymer and PEG- $b$-PBLG- $g$-PEG copolymer dissolved in $\mathrm{CDCl}_{3}$ take a-helix conformation $^{37}$.

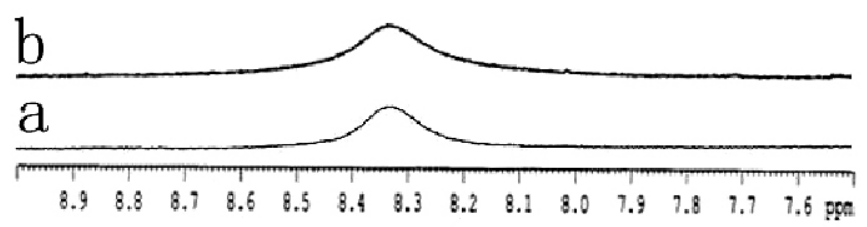

Figure 3. ${ }^{1} \mathrm{H}-\mathrm{NMR}$ spectra of (a) PBLG-graft-PEG and (b) PEG- $b$-PBLG$g$-PEG in $\mathrm{CDCl}_{3}$.

Chain conformation of polypeptide segments in the polypeptide copolymers in the solid state

Infrared (IR) spectra were measured for solid films of polypeptide copolymers cast from chloroform. Figure 4 presents the IR spectra of PBLGgraft-PEG copolymer (shown as curve a) and PEG- $b$-PBLG- $g$-PEG copolymer (shown as curve b). As it can be seen from Figure 4, the amide I and II bands of both PBLG-graft-PEG copolymer and PEG- $b$-PBLG- $g$-PEG copolymer appear at about $1653 \mathrm{~cm}^{-1}$ and $1548 \mathrm{~cm}^{-1}$, respectively, indicating that the polypeptide segments of the two polypeptide copolymers also exist in the a-helical conformation in the solid state ${ }^{38,39}$. This situation reveals that the chain conformations of polypeptide segments in both PBLG-graft-PEG and PEG- $b$ PBLG- $g$-PEG in the solid state are the same as that in PBLG homopolymer in solid state ${ }^{38,39}$



Figure 4. FTIR spectra of (a) PBLG-graft-PEG and (b) PEG-b-PBLG- $g$ PEG cast from chloroform.

Observations of transmission electron microscopy

Figure 5 presents the TEM photographs of (a) the micelles formed by PBLGgraft-PEG copolymer in ethanol and (b) the micelles formed by PEG- $b$-PBLG$g$-PEG in ethanol. As shown in Figure 5, both PBLG-graft-PEG copolymer and PEG- $b$-PBLG- $g$-PEG copolymer could self-assemble into polymeric micelles with the solvophobic PBLG segments aggregating as the inner core of the micelles surrounded by the solvophilic PEG chains. The morphology of the micelles formed by PBLG-graft-PEG in ethanol is thin spindle shape, while the morphology of most of the micelles formed by PEG- $b$-PBLG- $g$ PEG in ethanol is plump elliptical shape, only one spheroidal micelle found in the photograph could be a large aggregate. Compared with PBLG-graftPEG copolymer with pure graft structure, PEG- $b$-PBLG- $g$-PEG copolymer 
bears both block structure and graft structure. As described in document ${ }^{1}$, the difference in molecular architecture results in different morphologies of the micelles. This situation proves that the different morphologies of the micelles formed by PBLG-graft-PEG and PEG- $b$-PBLG- $g$-PEG in ethanol could be attributed to their different molecular structures.

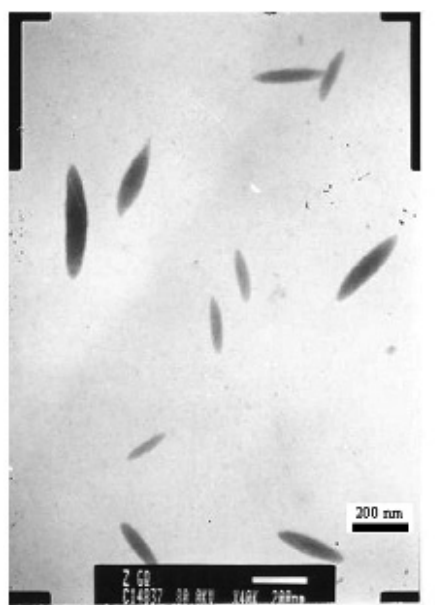

(a)

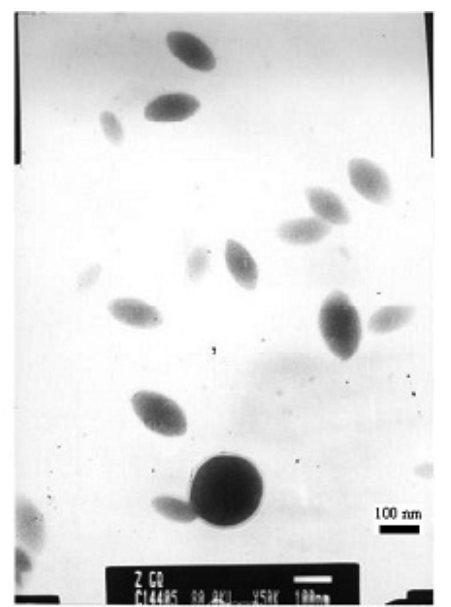

(b)
Figure 5. TEM photographs of (a) the micelles formed by PBLG-graftPEG in ethanol and (b) the micelles formed by PEG- $b$-PBLG- $g$-PEG in ethanol.

Effects of the introduction of PBLG homopolymer on the critical aggregation concentrations of PBLG-graft-PEG and PEG- $b$-PBLG- $g$ PEG

The critical aggregation concentrations of PBLG-graft-PEG or PEG- $b$ PBLG- $g$-PEG and their mixtures with PBLG homopolymer were confirmed according to the document ${ }^{14}$. Figure 6 shows the curves of the critical aggregation concentrations of PBLG-graft-PEG (shown by curve a) and PEG- $b$-PBLG- $g$-PEG (shown by curve b) in ethanol versus the content $(\mathrm{wt} \%)$ of PBLG homopolymer in the mixed system, where the testing temperature is $25{ }^{\circ} \mathrm{C}$. As shown in Figure 6, the critical aggregation concentrations of both PBLG-graft-PEG and PEG- $b$-PBLG- $g$-PEG decrease with the increase of PBLG homopolymer content in the mixed system, indicating that the PBLG homopolymer could promote the micellization of the two polypeptide copolymers. Due to the same solvophobic property, PBLG homopolymer could self-assemble into polymeric micelles together with PBLG-graft-PEG or PEG- $b$-PBLG- $g$-PEG through the interaction with PBLG segments in the two polypeptide copolymers ${ }^{1}$. Also seen from Figure 6, the decreasing degree of the critical aggregation concentration of PBLG-graft-PEG is larger than that of PEG- $b$-PBLG- $g$-PEG. As mentioned above, for the two copolymers, both PBLG segment length and the grafting ratio are similar, the main difference is their molecular structures. Because of the different molecular structures, the aggregating-resistance of PBLG homopolymer with polypeptide segments in PEG- $b$-PBLG- $g$-PEG is relatively bigger than that of PBLG homopolymer with polypeptide segments in PBLG-graft-PEG, suggesting that the difference of the decreasing degree of the critical aggregation concentrations of the two polypeptide copolymers in ethanol could be attributed to their different molecular structures.



Figure 6. Curves of the critical aggregation concentrations of PBLGgraft-PEG (shown by curve a) and PEG- $b$-PBLG- $g$-PEG (shown by curve $b$ ) in ethanol versus the content (wt\%) of PBLG homopolymer in the mixed system.

Effects of the testing temperature on the critical aggregation concentrations of PBLG-graft-PEG and PEG-b-PBLG-g-PEG

Figure 7 shows the curves of the critical aggregation concentrations of PBLG-graft-PEG (shown by curve a) and PEG- $b$-PBLG- $g$-PEG (shown by curve b) in ethanol versus the testing temperature. As it can be seen from Figure 7 , the critical aggregation concentrations of both PBLG-graft-PEG and PEG- $b$-PBLG- $g$-PEG in ethanol decrease with the increase of the testing temperature. As described in the documen $t^{40}$, the increase of the testing temperature promotes the interaction of PBLG segments in PBLG-graft-PEG or PEG- $b$-PBLG- $g$-PEG by accelerating the moving of polypeptide segments, suggesting the critical aggregation concentrations decrease with the increase of the testing temperature. Also seen from Figure 7, the decreasing degree of the critical aggregation concentrations of PBLG-graft-PEG is larger than that of PEG- $b$-PBLG-g-PEG. Owing to the different molecular structures, the moving-resistance of PBLG segments in PEG- $b$-PBLG- $g$-PEG is relatively bigger than that of polypeptide segments in PBLG-graft-PEG, indicating that their different molecular structures result in the difference of the decreasing degree of the critical aggregation concentrations.

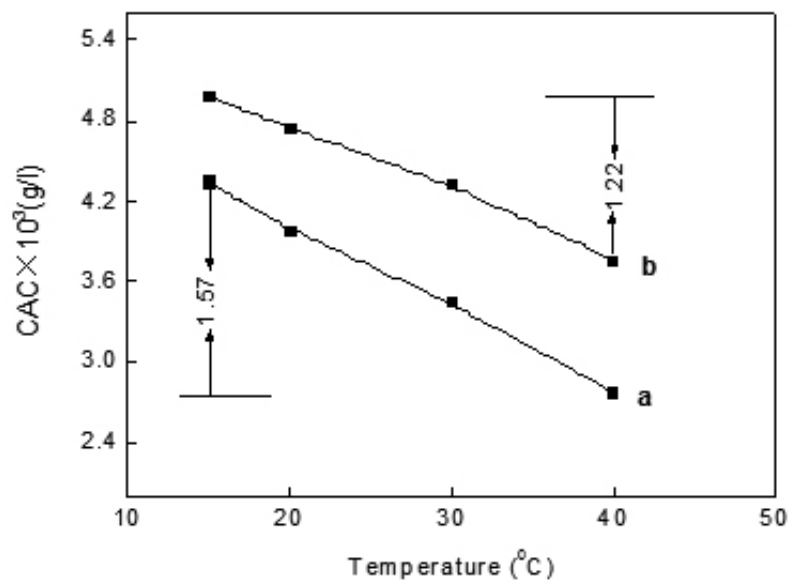

Figure 7. Curves of the critical aggregation concentrations of PBLGgraft-PEG (shown by curve a) and PEG- $b$-PBLG- $g$-PEG (shown by curve b) in ethanol versus the testing temperature.

\section{CONCLUSIONS}

Poly( $\gamma$-benzyl $L$-glutamate)-graft-poly(ethylene glycol) copolymer and poly(ethylene glycol)-block-poly( $\gamma$-benzyl $L$-glutamate)-graft-poly(ethylene glycol) copolymer have been synthesized. 'H-NMR analysis demonstrates 
that the polypeptide segments in both PBLG-graft-PEG and PEG- $b$-PBLG$g$-PEG dissolved in $\mathrm{CDCl}_{3}$ take a-helix conformation. FTIR measurements indicate that the polypeptide segments of both PBLG-graft-PEG and PEG- $b$ PBLG- $g$-PEG in the solid state also exist in the a-helical conformation. TEM observations show that both PBLG-graft-PEG copolymer and PEG- $b$-PBLG$g$-PEG copolymer could self-assemble to form core-shell structure micelles with different shapes in ethanol. Viscosity measurements reveal that both the increase of PBLG homopolymer content in the mixed system and the increase of the testing temperature promote the formation of the micelles of the two polypeptide copolymers.

\section{ACKNOWLEDGEMENT}

This work is supported by the Natural Science Foundation of Shandong Province (No. ZR2009FL019). Support from Shandong University of Technology (No. 4041 406015) is also appreciated.

\section{REFERENCES}

1. D.M. Tang, J.P. Lin, S.L. Lin, S.N. Zhang, T. Chen, X.H. Tian, Macromol. Rapid Commun. 25, 1241, (2004).

2. Z.S. Gao, A. Desjardins, A. Eisenberg, Macromolecules 25, 1300, (1992).

3. X.F. Zhong, S.K. Varshney, A. Eisenberg, Macromolecules 25, 7160, (1992).

4. J. Noolandi, K.M. Hong, Macromolecules 16, 1443, (1983).

5. T.M. Birshtein, E.B. Zhulina, Polymer 30, 170, (1989).

6. A. Rolland, J. O’Mullane, J. Goddard, L. Brookman, K. Petrak, J. Appl. Polym. Sci. 44, 1195, (1992).

7. M. Moffitt, A. Eisenberg, Macromolecules 30, 4363, (1997).

8. J.P. Lin, W.W. Ding, K.L. Hong, J.W. Mays, Z.D. Xu, Y.Z. Yuan, Soft Matter 4, 1605, (2008).

9. L.S. Zhang, J.P. Lin, S.L. Lin, J. Phys. Chem. B 111, 351, (2007).

10. L.S. Zhang, J.P. Lin, S.L. Lin, J. Phys. Chem. B 111, 9209, (2007).

11. W.W. Ding, S.L. Lin, J.P. Lin, L.S. Zhang, J. Phys. Chem. B 112, 776, (2008).

12. J.P. Lin, J.Q. Zhu, T. Chen, S.L. Lin, C.H. Cai, L.S. Zhang, Y. Zhuang, X.S. Wang, Biomaterials 30, 108, (2009)

13. W.Q. Zhang, L.Q. Shi, Y.L. An, K. Wu, L.C. Gao, Z. Liu, Macromolecules 37, 2924, (2004).

14. Z.S. Xu, L.X. Feng, J. Ji, S.Y. Cheng, Y.C. Chen, C.F. Yi, Eur. Polym. J. 34, $1499,(1998)$
15. T. Li, J.P. Lin, T. Chen, S.N. Zhang, Polymer 47, 4485, (2006).

16. G. Kwon, M. Naito, M. Yokoyama, T. Okano, Y. Sakurai, K. Kataoka, Langmuir 9, 945, (1993).

17. C.S. Cho, J.W. Nah, Y.I. Jeong, J.B. Cheon, S. Asayama, H. Ise, T. Akaike, Polymer 40, 6769, (1999).

18. A. Harada, S. Cammas, K. Kataoka, Macromolecules 29, 6183, (1996).

19. L.S. Zhang, J.P. Lin, S.L. Lin, Macromolecules 40, 5582, (2007).

20. J.P. Lin, S.N. Zhang, T. Chen, C.S. Liu, S.L. Lin, X.H. Tian, J. Biomed. Mater. Res. B 76, 432, (2006).

21. C.S. Cho, J.B. Cheon, Y.I. Jeong, I.S. Kim, S.H. Kim, T. Akaike, Macromol Rapid Commun. 18, 361, (1997).

22. I. Oh, K. Lee, H.Y. Kwon, Y.B. Lee, S.C. Shin, C.S. Cho, C.K. Kim, Int. J. Pharm. 181, 107, (1999).

23. P. Markland, G.L. Amidon, V.C. Yang, Int. J. Pharm. 178, 183, (1999).

24. Y.I. Jeong, J.W. Nah, H.C. Lee, S.H. Kim, C.S. Cho, Int. J. Pharm. 188, 49, (1999).

25. J.P. Lin, S.N. Zhang, T. Chen, S.L. Lin, H.T. Jin, Int. J. Pharm. 336, 49 , (2007).

26. J.P. Lin, G.Q. Zhu, X.M. Zhu, S.L. Lin, T. Nose, W.W. Ding, Polymer 49 $1132,(2008)$.

27. T. Chen, S.L. Lin, J.P. Lin, L.S. Zhang, Polymer 48, 2056, (2007)

28. J.B. Cheon, Y.I. Jeong, C.S. Cho, Polymer 40, 2041, (1999).

29. A. Harada, K. Kataoka, Macromolecules 28, 5294, (1995).

30. N. Higashi, J. Kawahara, M. Niwa, J. Colloid Interf. Sci. 288, 83, (2005).

31. J.W. Nah, Y.I. Jeong, C.S. Cho, J. Polym. Sci. Pol. Phys. 36, 415, (1998).

32. J.P. Lin, N. Liu, J. Chen, D.F. Zhou, Polymer 41, 6189, (2000).

33. J.P. Lin, A. Abe, H. Furuya, S. Okamoto, Macromolecules 29, 2584, (1996).

34. A. Abe, T. Yamazaki, Macromolecules 22, 2138, (1989).

35. K. Inomata, N. Ohara, H. Shimizu, T. Nose, Polymer 39, 3379, (1998).

36. K. Inomata, H. Shimizu, T. Nose, J. Polym. Sci. Pol. Phys. 38, 1331, (2000).

37. J.A. Ferretti, B.W. Ninham, Macromolecules 3, 30, (1970)

38. N. Liu, J.P. Lin, T. Chen, J.D. Chen, D.F. Zhou, L. Li, Polym. J. 33, 898, (2001).

39. C.S. Cho, Y.I. Jeong, S.H. Kim, J.W. Nah, M. Kubota, T. Komota, Polymer 41, 5185, (2000)

40. C. Price, K.D. Kendall, R.B. Stubbersfield, B. Wright, Polym. Commun. 24, 326, (1983) 